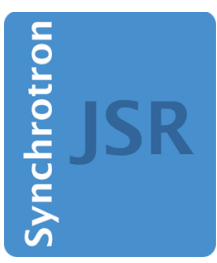

JOURNAL OF

SYNCHROTRON

RADIATION

Volume 24 (2017)

Supporting information for article:

Uridine as a new scavenger for synchrotron-based structural biology techniques

Eva Crosas, Albert Castellvi, Isidro Crespo, Daniel Fulla, Fernando Gil-Ortiz, Gustavo Fuertes, Christina S. Kamma-Lorger, Marc Malfois, Miguel A. G. Aranda and Jordi Juanhuix 


\section{S1. MX results}

Table S1 Crystal definition and classification according to the concentration of uridine, the beam size dimensions, dose rate and temperature. Crystals 12 and 16 were used to evaluate site-specific radiation damage.

\begin{tabular}{lllll}
\hline $\begin{array}{l}\text { Crystal } \\
\text { Number }\end{array}$ & [Uridine] $(\mathrm{mM})$ & $\begin{array}{l}\text { Beam size } \\
\text { FWHM }(\mathrm{h} x \mathrm{v} \mu \mathrm{m})\end{array}$ & Dose Rate $\left(\mathrm{kGy} \mathrm{s}^{-1}\right)$ & $\mathrm{T}(\mathrm{K})$ \\
\hline $1-4$ & 0 & $100 \times 85$ & 13.8 & $\mathrm{RT}$ \\
$5-8$ & 200 & $100 \times 85$ & 13.8 & $\mathrm{RT}$ \\
$9-11$ & 500 & $100 \times 85$ & 13.8 & $\mathrm{RT}$ \\
$12-15$ & 0 & $90 \times 80$ & 20.0 & RT \\
$16-18$ & 1000 & $90 \times 80$ & 20.0 & RT \\
$19-21$ & 0 & $86 \times 78$ & 90.0 & 100 \\
22 & 500 & $86 \times 78$ & 90.0 & 100 \\
$23-25$ & 1000 & $86 \times 78$ & 90.0 & 100 \\
\hline
\end{tabular}

Table S2 Data collection statistics for all crystals collected at room temperature extracted from the initial integration by XDS. Resolution range is 50-1.7 $\AA$. Space group for all crystals is $\mathrm{P}_{4} 2_{1} 2$.

Crystals 12 and 16 are used in the main text to evaluate the site-specific radiation damage.

Crystal 1 (No uridine)

\begin{tabular}{|c|c|c|c|c|c|c|c|c|c|c|}
\hline $\begin{array}{l}\begin{array}{l}\text { Dose } \\
\text { (MGy) }\end{array} \\
\end{array}$ & $\begin{array}{l}\text { Unique } \\
\text { reflections }\end{array}$ & $\begin{array}{l}\text { Complete } \\
\text {-ness }(\%) \\
\end{array}$ & $\langle\mathrm{I}\rangle / \sigma(\mathrm{I})$ & $\begin{array}{l}\text { R-meas } \\
(\%)\end{array}$ & $\begin{array}{l}\begin{array}{l}\text { B-factor } \\
\left(\AA^{2}\right)\end{array} \\
\end{array}$ & $\begin{array}{l}\mathrm{a}=\mathrm{b} \\
(\AA)\end{array}$ & $\begin{array}{l}\mathrm{c} \\
(\AA)\end{array}$ & $\begin{array}{l}\begin{array}{l}\text { Mosaicity } \\
\text { (deg) }\end{array} \\
\end{array}$ & $\mathrm{CC} 1 / 2$ & $\mathrm{I}_{\mathrm{n}} / \mathrm{I}_{0}$ \\
\hline 0.31 & 8495 & $\begin{array}{l}98.9 \\
(99.5)\end{array}$ & $\begin{array}{l}30.76 \\
(15.03)\end{array}$ & $\begin{array}{l}4.5 \\
(11.1)\end{array}$ & 23.15 & 79.2 & 37.9 & 0.034 & 99.9 & 1.00 \\
\hline 0.62 & 8508 & $\begin{array}{l}99.0 \\
(99.5)\end{array}$ & $\begin{array}{l}21.22 \\
(8.48)\end{array}$ & $\begin{array}{l}6.1 \\
(21.4)\end{array}$ & 26.33 & 79.3 & 37.9 & 0.106 & 99.9 & 0.86 \\
\hline 0.93 & 8492 & $\begin{array}{l}98.8 \\
(99.2)\end{array}$ & $\begin{array}{l}21.12 \\
(6.33)\end{array}$ & $\begin{array}{l}6.2 \\
(30.7)\end{array}$ & 30.28 & 79.3 & 37.9 & 0.085 & 99.9 & 0.64 \\
\hline 1.24 & 8480 & $\begin{array}{l}98.8 \\
(99.4)\end{array}$ & $\begin{array}{l}15.39 \\
(2.08)\end{array}$ & $\begin{array}{l}9.2 \\
(90.6)\end{array}$ & 37.24 & 79.3 & 37.8 & 0.096 & 99.9 & 0.36 \\
\hline 1.55 & 8484 & $\begin{array}{l}99.0 \\
(99.0)\end{array}$ & $\begin{array}{l}6.27 \\
(0.33)\end{array}$ & $\begin{array}{l}27.9 \\
(520.4)\end{array}$ & 44.99 & 79.3 & 37.8 & 0.184 & 99.4 & 0.14 \\
\hline
\end{tabular}

Crystal 2 (No uridine)

\begin{tabular}{lllllllllll}
\hline $\begin{array}{l}\text { Dose } \\
(\mathrm{MGy})\end{array}$ & $\begin{array}{l}\text { Unique } \\
\text { reflections }\end{array}$ & $\begin{array}{l}\text { Complete } \\
\text {-ness }(\%)\end{array}$ & $\langle\mathrm{I}>/ \sigma(\mathrm{I})$ & $\begin{array}{l}\mathrm{R} \text {-meas } \\
(\%)\end{array}$ & $\begin{array}{l}\mathrm{B} \text {-factor } \\
\left(\AA^{2}\right)\end{array}$ & $\begin{array}{l}\mathrm{a}=\mathrm{b} \\
(\AA)\end{array}$ & $\begin{array}{l}\mathrm{c} \\
(\AA)\end{array}$ & $\begin{array}{l}\text { Mosaicity } \\
(\mathrm{deg})\end{array}$ & $\mathrm{CC} 1 / 2$ & $\mathrm{I}_{\mathrm{n}} / \mathrm{I}_{0}$ \\
\hline & & & & & & & & & & \\
0.31 & 8559 & $\begin{array}{l}99.6 \\
(99.9)\end{array}$ & $\begin{array}{l}35.66 \\
(19.06)\end{array}$ & $\begin{array}{l}3.8 \\
(8.8)\end{array}$ & 23.12 & 79.2 & 37.9 & 0.033 & 99.9 & 1.00
\end{tabular}




\begin{tabular}{lllllllllll}
0.62 & 8564 & 99.6 & 33.48 & 4.0 & 25.68 & 79.3 & 37.9 & 0.033 & 99.9 & 0.86 \\
& & $(99.8)$ & $(15.42)$ & $(11.1)$ & & & & & & \\
0.93 & 8569 & 99.7 & 30.43 & 4.3 & 29.71 & 79.3 & 37.9 & 0.041 & 99.9 & 0.66 \\
& & $(99.9)$ & $(10.7)$ & $(16.3)$ & & & & & & \\
1.24 & 8564 & 99.8 & 22.01 & 6.0 & 35.84 & 79.3 & 37.9 & 0.067 & 99.9 & 0.41 \\
& & $(99.9)$ & $(4.29)$ & $(45.3)$ & & & & & & \\
1.55 & 8538 & 99.7 & 11.56 & 15.9 & 40.96 & 79.3 & 37.8 & 0.147 & 99.8 & 0.21 \\
& & $(99.6)$ & $(0.84)$ & $(239.5)$ & & & & & & \\
\hline
\end{tabular}

Crystal 3 (No uridine)

\begin{tabular}{|c|c|c|c|c|c|c|c|c|c|c|}
\hline $\begin{array}{l}\text { Dose } \\
\text { (MGy) }\end{array}$ & $\begin{array}{l}\text { Unique } \\
\text { reflections }\end{array}$ & $\begin{array}{l}\text { Complete } \\
\text {-ness }(\%)\end{array}$ & $\langle\mathrm{I}\rangle / \sigma(\mathrm{I})$ & $\begin{array}{l}\text { R-meas } \\
(\%)\end{array}$ & $\begin{array}{l}\text { B-factor } \\
\left(\AA^{2}\right)\end{array}$ & $\begin{array}{l}a=b \\
(\AA)\end{array}$ & $\begin{array}{l}\mathrm{c} \\
(\AA)\end{array}$ & $\begin{array}{l}\text { Mosaicity } \\
\text { (deg) }\end{array}$ & $\mathrm{CC} 1 / 2$ & $\mathrm{I}_{\mathrm{n}} / \mathrm{I}_{0}$ \\
\hline 0.31 & 8564 & $\begin{array}{l}99.8 \\
(99.8)\end{array}$ & $\begin{array}{l}39.44 \\
(19.00)\end{array}$ & $\begin{array}{l}3.4 \\
(8.6)\end{array}$ & 24.14 & 79.2 & 37.9 & 0.054 & 99.9 & 1.00 \\
\hline 0.62 & 8568 & $\begin{array}{l}99.7 \\
(99.4)\end{array}$ & $\begin{array}{l}36.03 \\
(14.52)\end{array}$ & $\begin{array}{l}3.6 \\
(11.5)\end{array}$ & 27.67 & 79.2 & 37.9 & 0.057 & 100.0 & 0.80 \\
\hline 0.93 & 8575 & $\begin{array}{l}99.8 \\
(99.9)\end{array}$ & $\begin{array}{l}28.21 \\
(7.02)\end{array}$ & $\begin{array}{l}4.7 \\
(30.1)\end{array}$ & 33.91 & 79.3 & 37.9 & 0.072 & 100.0 & 0.54 \\
\hline 1.24 & 8567 & $\begin{array}{l}99.8 \\
(99.6)\end{array}$ & $\begin{array}{l}14.39 \\
(1.06)\end{array}$ & $\begin{array}{l}11.8 \\
(200.8)\end{array}$ & 43.80 & 79.4 & 37.8 & 0.148 & 99.9 & 0.26 \\
\hline 1.55 & 8300 & $\begin{array}{l}97.2 \\
(92.0)\end{array}$ & $\begin{array}{l}5.37 \\
(-)\end{array}$ & $\begin{array}{l}45.8 \\
(-)\end{array}$ & 52.79 & 79.4 & 37.8 & 0.270 & 99.0 & 0.09 \\
\hline
\end{tabular}

Crystal 4 (No uridine)

\begin{tabular}{|c|c|c|c|c|c|c|c|c|c|c|}
\hline $\begin{array}{l}\begin{array}{l}\text { Dose } \\
(\mathrm{MGy})\end{array} \\
\end{array}$ & $\begin{array}{l}\text { Unique } \\
\text { reflections }\end{array}$ & $\begin{array}{l}\text { Complete } \\
\text {-ness }(\%)\end{array}$ & $\langle\mathrm{I}\rangle / \sigma(\mathrm{I})$ & $\begin{array}{l}\text { R-meas } \\
(\%)\end{array}$ & $\begin{array}{l}\text { B-factor } \\
\left(\AA^{2}\right)\end{array}$ & $\begin{array}{l}\mathrm{a}=\mathrm{b} \\
(\AA)\end{array}$ & $\begin{array}{l}\mathrm{c} \\
(\AA)\end{array}$ & $\begin{array}{l}\text { Mosaicity } \\
\text { (deg) }\end{array}$ & $\mathrm{CC} 1 / 2$ & $\mathrm{I}_{\mathrm{n}} / \mathrm{I}_{0}$ \\
\hline 0.62 & 8569 & $\begin{array}{l}99.7 \\
(100)\end{array}$ & $\begin{array}{l}30.04 \\
(12.81)\end{array}$ & $\begin{array}{l}4.4 \\
(13.1)\end{array}$ & 26.33 & 79.3 & 37.9 & 0.037 & 99.9 & 0.84 \\
\hline 0.93 & 8577 & $\begin{array}{l}99.7 \\
(99.9)\end{array}$ & $\begin{array}{l}25.68 \\
(8.59)\end{array}$ & $\begin{array}{l}5.0 \\
(20.8)\end{array}$ & 30.64 & 79.3 & 37.9 & 0.039 & 99.9 & 0.62 \\
\hline 1.24 & 8589 & $\begin{array}{l}99.7 \\
(99.5)\end{array}$ & $\begin{array}{l}18.32 \\
(3.07)\end{array}$ & $\begin{array}{l}7.2 \\
(68.4)\end{array}$ & 38.45 & 79.4 & 37.9 & 0.054 & 99.9 & 0.36 \\
\hline 1.55 & 8596 & $\begin{array}{l}99.7 \\
(99.0)\end{array}$ & $\begin{array}{l}8.00 \\
(0.34)\end{array}$ & $\begin{array}{l}20.3 \\
(512.6)\end{array}$ & 50.12 & 79.5 & 37.9 & 0.107 & 99.8 & 0.13 \\
\hline
\end{tabular}

Crystal 5 (200mM uridine)

\begin{tabular}{|c|c|c|c|c|c|c|c|c|c|c|}
\hline $\begin{array}{l}\text { Dose } \\
\text { (MGy) }\end{array}$ & $\begin{array}{l}\text { Unique } \\
\text { reflections }\end{array}$ & $\begin{array}{l}\text { Complete } \\
\text {-ness }(\%)\end{array}$ & $\langle\mathrm{I}\rangle / \sigma(\mathrm{I})$ & $\begin{array}{l}\text { R-meas } \\
(\%)\end{array}$ & $\begin{array}{l}\text { B-factor } \\
(\AA 2)\end{array}$ & $\begin{array}{l}a=b \\
(\AA)\end{array}$ & $\begin{array}{l}\mathrm{c} \\
(\AA)\end{array}$ & $\begin{array}{l}\text { Mosaicity } \\
\text { (deg) }\end{array}$ & $\mathrm{CC} 1 / 2$ & $\mathrm{I}_{\mathrm{n}} / \mathrm{I}_{0}$ \\
\hline 0.31 & 8329 & $\begin{array}{l}97.2 \\
(98.7)\end{array}$ & $\begin{array}{l}34.08 \\
(16.16)\end{array}$ & $\begin{array}{l}3.8 \\
(10.3)\end{array}$ & 24.29 & 79.1 & 37.9 & 0.034 & 99.9 & 1.00 \\
\hline 0.62 & 8334 & $\begin{array}{l}97.2 \\
(98.3)\end{array}$ & $\begin{array}{l}32.18 \\
(13.72)\end{array}$ & $\begin{array}{l}4.0 \\
(12.1)\end{array}$ & 26.41 & 79.2 & 37.9 & 0.036 & 99.9 & 0.88 \\
\hline 0.93 & 8332 & $\begin{array}{l}97.2 \\
(98.8)\end{array}$ & $\begin{array}{l}29.41 \\
(10.30)\end{array}$ & $\begin{array}{l}4.3 \\
(15.9)\end{array}$ & 29.55 & 79.2 & 37.9 & 0.041 & 99.9 & 0.72 \\
\hline 1.24 & 8336 & $\begin{array}{l}97.1 \\
(97.5)\end{array}$ & $\begin{array}{l}23.71 \\
(6.37)\end{array}$ & $\begin{array}{l}5.1 \\
(28.6)\end{array}$ & 34.05 & 79.3 & 37.9 & 0.049 & 99.9 & 0.52 \\
\hline 1.55 & 8351 & $\begin{array}{l}97.3 \\
(98.5)\end{array}$ & $\begin{array}{l}16.6 \\
(2.09)\end{array}$ & $\begin{array}{l}7.7 \\
(93.1)\end{array}$ & 41.62 & 79.3 & 37.9 & 0.073 & 99.9 & 0.31 \\
\hline 1.86 & 8305 & $\begin{array}{l}96.7 \\
(94.9)\end{array}$ & $\begin{array}{l}7.96 \\
(0.13)\end{array}$ & $\begin{array}{l}20.8 \\
(1134)\end{array}$ & 54.50 & 79.4 & 37.8 & 0.145 & 99.8 & 0.12 \\
\hline
\end{tabular}

Crystal 6 (200mM uridine)

\begin{tabular}{lllllllllll}
\hline $\begin{array}{l}\text { Dose } \\
(\mathrm{MGy})\end{array}$ & $\begin{array}{l}\text { Unique } \\
\text { reflections }\end{array}$ & $\begin{array}{l}\text { Complete } \\
- \text {-ness }(\%)\end{array}$ & $<\mathrm{I}>/ \sigma(\mathrm{I})$ & $\begin{array}{l}\mathrm{R}-\text {-meas } \\
(\%)\end{array}$ & $\begin{array}{l}\mathrm{B} \text {-factor } \\
(\AA 2)\end{array}$ & $\begin{array}{l}\mathrm{a}=\mathrm{b} \\
(\AA)\end{array}$ & $\begin{array}{l}\mathrm{c} \\
(\AA)\end{array}$ & $\begin{array}{l}\text { Mosaicity } \\
(\mathrm{deg})\end{array}$ & $\mathrm{CC} 1 / 2$ & $\mathrm{I}_{\mathrm{n}} / \mathrm{I}_{0}$ \\
\hline
\end{tabular}




\begin{tabular}{|c|c|c|c|c|c|c|c|c|c|c|}
\hline 0.31 & 8535 & $\begin{array}{l}99.5 \\
(99.0)\end{array}$ & $\begin{array}{l}36.25 \\
(18.51)\end{array}$ & $\begin{array}{l}3.7 \\
(9.2)\end{array}$ & 23.73 & 79.2 & 37.9 & 0.039 & 99.9 & 1.00 \\
\hline 0.62 & 8539 & $\begin{array}{l}99.4 \\
(98.8)\end{array}$ & $\begin{array}{l}34.22 \\
(15.42)\end{array}$ & $\begin{array}{l}3.8 \\
(10.9)\end{array}$ & 26.14 & 79.2 & 37.9 & 0.044 & 99.9 & 0.89 \\
\hline 0.93 & 8539 & $\begin{array}{l}99.5 \\
(98.9)\end{array}$ & $\begin{array}{l}32.71 \\
(11.32)\end{array}$ & $\begin{array}{l}3.9 \\
(15.4)\end{array}$ & 29.61 & 79.3 & 37.9 & 0.054 & 100.0 & 0.76 \\
\hline 1.24 & 8539 & $\begin{array}{l}99.5 \\
(98.7)\end{array}$ & $\begin{array}{l}28.64 \\
(6.04)\end{array}$ & $\begin{array}{l}4.6 \\
(30.8)\end{array}$ & 33.96 & 79.3 & 37.9 & 0.079 & 100.0 & 0.58 \\
\hline 1.55 & 8537 & $\begin{array}{l}99.5 \\
(99.0)\end{array}$ & $\begin{array}{l}19.27 \\
(1.98)\end{array}$ & $\begin{array}{l}8.2 \\
(100.0)\end{array}$ & 39.00 & 79.4 & 37.9 & 0.132 & 99.9 & 0.38 \\
\hline 1.86 & 8575 & $\begin{array}{l}99.5 \\
(98.7)\end{array}$ & $\begin{array}{l}10.38 \\
(0.54)\end{array}$ & $\begin{array}{l}19.0 \\
(345.5)\end{array}$ & 43.41 & 79.4 & 37.9 & 0.192 & 99.7 & 0.22 \\
\hline
\end{tabular}

Crystal 7 (200mM uridine)

\begin{tabular}{|c|c|c|c|c|c|c|c|c|c|c|}
\hline $\begin{array}{l}\begin{array}{l}\text { Dose } \\
\text { (MGy) }\end{array} \\
\end{array}$ & $\begin{array}{l}\text { Unique } \\
\text { reflections }\end{array}$ & $\begin{array}{l}\text { Complete } \\
\text {-ness (\%) }\end{array}$ & $\langle\mathrm{I}\rangle / \sigma(\mathrm{I})$ & $\begin{array}{l}\text { R-meas } \\
(\%)\end{array}$ & $\begin{array}{l}\text { B-factor } \\
(\AA 2)\end{array}$ & $\begin{array}{l}a=b \\
(\AA)\end{array}$ & $\begin{array}{l}\mathrm{c} \\
(\AA)\end{array}$ & $\begin{array}{l}\text { Mosaicity } \\
\text { (deg) }\end{array}$ & $\mathrm{CC} 1 / 2$ & $\mathrm{I}_{\mathrm{n}} / \mathrm{I}_{0}$ \\
\hline 0.31 & 8541 & $\begin{array}{l}99.7 \\
(99.9)\end{array}$ & $\begin{array}{l}30.78 \\
(13.4)\end{array}$ & $\begin{array}{l}4.4 \\
(12.1)\end{array}$ & 24.49 & 79.1 & 38.0 & 0.043 & 99.9 & 1.00 \\
\hline 0.62 & 8553 & $\begin{array}{l}99.8 \\
(99.9)\end{array}$ & $\begin{array}{l}28.78 \\
(11.5)\end{array}$ & $\begin{array}{l}4.7 \\
(14.6)\end{array}$ & 26.46 & 79.1 & 37.9 & 0.041 & 99.9 & 0.88 \\
\hline 0.93 & 8565 & $\begin{array}{l}99.8 \\
(99.9)\end{array}$ & $\begin{array}{l}26.25 \\
(8.97)\end{array}$ & $\begin{array}{l}5.0 \\
(19.1)\end{array}$ & 29.11 & 79.2 & 37.9 & 0.042 & 99.9 & 0.74 \\
\hline 1.24 & 8582 & $\begin{array}{l}99.9 \\
(99.5)\end{array}$ & $\begin{array}{l}22.52 \\
(5.55)\end{array}$ & $\begin{array}{l}5.8 \\
(33.0)\end{array}$ & 33.46 & 79.2 & 37.9 & 0.047 & 99.9 & 0.55 \\
\hline 1.55 & 8594 & $\begin{array}{l}99.8 \\
(99.4)\end{array}$ & $\begin{array}{l}15.97 \\
(1.82)\end{array}$ & $\begin{array}{l}8.5 \\
(112.3)\end{array}$ & 41.48 & 79.3 & 37.9 & 0.063 & 99.9 & 0.33 \\
\hline 1.86 & 8597 & $\begin{array}{l}99.7 \\
(99.3)\end{array}$ & $\begin{array}{l}7.8 \\
(0.16)\end{array}$ & $\begin{array}{l}23.6 \\
(954.9)\end{array}$ & 54.46 & 79.5 & 37.8 & 0.102 & 99.7 & 0.13 \\
\hline
\end{tabular}

Crystal 8 (200mM uridine)

\begin{tabular}{|c|c|c|c|c|c|c|c|c|c|c|}
\hline $\begin{array}{l}\text { Dose } \\
\text { (MGy) }\end{array}$ & $\begin{array}{l}\text { Unique } \\
\text { reflections }\end{array}$ & $\begin{array}{l}\text { Complete } \\
\text {-ness (\%) }\end{array}$ & $\langle\mathrm{I}\rangle / \sigma(\mathrm{I})$ & $\begin{array}{l}\text { R-meas } \\
(\%)\end{array}$ & $\begin{array}{l}\text { B-factor } \\
(\AA 2)\end{array}$ & $\begin{array}{l}a=b \\
(\AA)\end{array}$ & $\begin{array}{l}\mathrm{c} \\
(\AA)\end{array}$ & $\begin{array}{l}\text { Mosaicity } \\
\text { (deg) }\end{array}$ & $\mathrm{CC} 1 / 2$ & $\mathrm{I}_{\mathrm{n}} / \mathrm{I}_{0}$ \\
\hline 0.31 & 8452 & $\begin{array}{l}98.6 \\
(99.3)\end{array}$ & $\begin{array}{l}31.2 \\
(16.78)\end{array}$ & $\begin{array}{l}4.3 \\
(9.9)\end{array}$ & 23.40 & 79.1 & 37.96 & 0.026 & 99.9 & 1.00 \\
\hline 0.62 & 8460 & $\begin{array}{l}98.6 \\
(99.2)\end{array}$ & $\begin{array}{l}29.3 \\
(14.4)\end{array}$ & $\begin{array}{l}4.5 \\
(11.5)\end{array}$ & 25.21 & 79.1 & 37.95 & 0.025 & 99.9 & 0.88 \\
\hline 0.93 & 8463 & $\begin{array}{l}98.6 \\
(99.1)\end{array}$ & $\begin{array}{l}27.54 \\
(11.27)\end{array}$ & $\begin{array}{l}4.6 \\
(15.2)\end{array}$ & 28.25 & 79.17 & 37.94 & 0.027 & 99.9 & 0.74 \\
\hline 1.24 & 8485 & $\begin{array}{l}98.8 \\
(98.7)\end{array}$ & $\begin{array}{l}24.32 \\
(7.00)\end{array}$ & $\begin{array}{l}5.1 \\
(25.5)\end{array}$ & 33.20 & 79.24 & 37.93 & 0.04 & 99.9 & 0.57 \\
\hline 1.55 & 8491 & $\begin{array}{l}98.7 \\
(99.2)\end{array}$ & $\begin{array}{l}17.34 \\
(2.17)\end{array}$ & $\begin{array}{l}7.5 \\
(90.8)\end{array}$ & 41.78 & 79.34 & 37.92 & 0.068 & 99.9 & 0.34 \\
\hline 1.86 & 8502 & $\begin{array}{l}98.5 \\
(98.1)\end{array}$ & $\begin{array}{l}8.84 \\
(0.22)\end{array}$ & $\begin{array}{l}18.3 \\
(647.9)\end{array}$ & 53.81 & 79.46 & 37.90 & 0.111 & 99.8 & 0.14 \\
\hline
\end{tabular}

Crystal 9 (500mM uridine)

\begin{tabular}{|c|c|c|c|c|c|c|c|c|c|c|}
\hline $\begin{array}{l}\text { Dose } \\
\text { (MGy) }\end{array}$ & $\begin{array}{l}\text { Unique } \\
\text { reflections }\end{array}$ & $\begin{array}{l}\text { Complete } \\
\text {-ness }(\%)\end{array}$ & $\langle\mathrm{I}\rangle / \sigma(\mathrm{I})$ & $\begin{array}{l}\text { R-meas } \\
(\%)\end{array}$ & $\begin{array}{l}\text { B-factor } \\
(\AA 2)\end{array}$ & $\begin{array}{l}a=b \\
(\AA)\end{array}$ & $\begin{array}{l}\mathrm{c} \\
(\AA)\end{array}$ & $\begin{array}{l}\text { Mosaicity } \\
\text { (deg) }\end{array}$ & $\mathrm{CC} 1 / 2$ & $\mathrm{I}_{\mathrm{n}} / \mathrm{I}_{0}$ \\
\hline 0.31 & 8337 & $\begin{array}{l}97.3 \\
(98.8)\end{array}$ & $\begin{array}{l}35.49 \\
(18.22)\end{array}$ & $\begin{array}{l}3.7 \\
(9.0)\end{array}$ & 23.88 & 79.10 & 37.98 & 0.039 & 99.9 & 1.00 \\
\hline 0.62 & 8345 & $\begin{array}{l}97.3 \\
(98.7)\end{array}$ & $\begin{array}{l}33.61 \\
(16.13)\end{array}$ & $\begin{array}{l}3.8 \\
(10.2)\end{array}$ & 25.39 & 79.13 & 37.97 & 0.037 & 99.9 & 0.90 \\
\hline 0.93 & 8366 & $\begin{array}{l}97.5 \\
(99.0)\end{array}$ & $\begin{array}{l}31.82 \\
(13.59)\end{array}$ & $\begin{array}{l}3.9 \\
(12.1)\end{array}$ & 27.65 & 79.18 & 37.97 & 0.039 & 99.9 & 0.80 \\
\hline 1.24 & 8365 & $\begin{array}{l}97.4 \\
(98.7)\end{array}$ & $\begin{array}{l}30.35 \\
(10.4)\end{array}$ & $4(16.0)$ & 30.74 & 79.23 & 37.97 & 0.041 & 99.9 & 0.68 \\
\hline 1.55 & 8391 & $\begin{array}{l}97.5 \\
(99.0)\end{array}$ & $\begin{array}{l}26.57 \\
(6.39)\end{array}$ & $\begin{array}{l}4.5 \\
(27.6)\end{array}$ & 35.18 & 79.28 & 37.97 & 0.052 & 100 & 0.53 \\
\hline
\end{tabular}




\begin{tabular}{lllllllllll}
1.86 & 8387 & 97.5 & 20.06 & 6.2 & 41.61 & 79.33 & 37.97 & 0.073 & 99.9 & 0.35 \\
& & $(98.9)$ & $(2.48)$ & $(76.4)$ & & & & & & \\
2.17 & 8348 & 97.5 & 11.64 & 13.9 & 47.10 & 79.34 & 37.96 & 0.123 & 99.8 & 0.19 \\
& & $(98.9)$ & $(0.61)$ & $(296.3)$ & & & & & & \\
\hline
\end{tabular}

Crystal 10 (500mM uridine)

\begin{tabular}{|c|c|c|c|c|c|c|c|c|c|c|}
\hline $\begin{array}{l}\begin{array}{l}\text { Dose } \\
\text { (MGy) }\end{array} \\
\end{array}$ & $\begin{array}{l}\text { Unique } \\
\text { reflections }\end{array}$ & $\begin{array}{l}\text { Complete } \\
\text {-ness (\%) }\end{array}$ & $\langle\mathrm{I}\rangle / \sigma(\mathrm{I})$ & $\begin{array}{l}\text { R-meas } \\
(\%)\end{array}$ & $\begin{array}{l}\text { B-factor } \\
(\AA 2)\end{array}$ & $\begin{array}{l}\mathrm{a}=\mathrm{b} \\
(\AA)\end{array}$ & $\begin{array}{l}\mathrm{c} \\
(\AA)\end{array}$ & $\begin{array}{l}\text { Mosaicity } \\
\text { (deg) }\end{array}$ & $\mathrm{CC} 1 / 2$ & $\mathrm{I}_{\mathrm{n}} / \mathrm{I}_{0}$ \\
\hline 0.31 & 8551 & $\begin{array}{l}99.8 \\
(99.9)\end{array}$ & $\begin{array}{l}38.18 \\
(21.88)\end{array}$ & $\begin{array}{l}3.6 \\
(7.9)\end{array}$ & 23.53 & 79.1 & 38.0 & 0.048 & 99.9 & 1.00 \\
\hline 0.62 & 8551 & $\begin{array}{l}99.8 \\
(99.7)\end{array}$ & $\begin{array}{l}36.67 \\
(19.18)\end{array}$ & $\begin{array}{l}3.6 \\
(9.1)\end{array}$ & 25.36 & 79.1 & 38.0 & 0.049 & 99.9 & 0.92 \\
\hline 0.93 & 8559 & $\begin{array}{l}99.8 \\
(99.6)\end{array}$ & $\begin{array}{l}36.27 \\
(16.37)\end{array}$ & $\begin{array}{l}3.6 \\
(10.4)\end{array}$ & 27.48 & 79.1 & 38.0 & 0.05 & 99.9 & 0.82 \\
\hline 1.24 & 8565 & $\begin{array}{l}99.8 \\
(99.8)\end{array}$ & $\begin{array}{l}32.81 \\
(12.89)\end{array}$ & $\begin{array}{l}3.8 \\
(13.1)\end{array}$ & 30.26 & 79.2 & 38.0 & 0.055 & 99.9 & 0.71 \\
\hline 1.55 & 8576 & $\begin{array}{l}99.8 \\
(99.7)\end{array}$ & $\begin{array}{l}31.76 \\
(9.11)\end{array}$ & $\begin{array}{l}3.8 \\
(19.6)\end{array}$ & 34.05 & 79.2 & 38.0 & 0.06 & 100 & 0.58 \\
\hline 1.86 & 8585 & $\begin{array}{l}99.8 \\
(99.6)\end{array}$ & $\begin{array}{l}26.11 \\
(4.36)\end{array}$ & $\begin{array}{l}4.8 \\
(46.0)\end{array}$ & 39.36 & 79.3 & 38.0 & 0.08 & 100 & 0.41 \\
\hline 2.17 & 8588 & $\begin{array}{l}99.8 \\
(99.5)\end{array}$ & $\begin{array}{l}14.09 \\
(1.12)\end{array}$ & $\begin{array}{l}12.0 \\
(189.6)\end{array}$ & 43.21 & 79.2 & 37.9 & 0.148 & 99.9 & 0.25 \\
\hline
\end{tabular}

Crystal 11 (500mM uridine)

\begin{tabular}{|c|c|c|c|c|c|c|c|c|c|c|}
\hline $\begin{array}{l}\text { Dose } \\
\text { (MGy) }\end{array}$ & $\begin{array}{l}\text { Unique } \\
\text { reflections }\end{array}$ & $\begin{array}{l}\text { Complete } \\
\text {-ness (\%) }\end{array}$ & $\langle\mathrm{I}\rangle / \sigma(\mathrm{I})$ & $\begin{array}{l}\text { R-meas } \\
(\%)\end{array}$ & $\begin{array}{l}\text { B-factor } \\
(\AA 2)\end{array}$ & $\begin{array}{l}\mathrm{a}=\mathrm{b} \\
(\AA)\end{array}$ & $\begin{array}{l}\mathrm{c} \\
(\AA)\end{array}$ & $\begin{array}{l}\text { Mosaicity } \\
(\mathrm{deg})\end{array}$ & $\mathrm{CC} 1 / 2$ & $\mathrm{I}_{\mathrm{n}} / \mathrm{I}_{0}$ \\
\hline 0.31 & 8538 & $\begin{array}{l}99.9 \\
(99.7)\end{array}$ & $\begin{array}{l}29.31 \\
(9.98)\end{array}$ & $\begin{array}{l}4.9 \\
(19.0)\end{array}$ & 26.03 & 79.1 & 37.9 & 0.159 & 99.9 & 1.00 \\
\hline 0.62 & 8541 & $\begin{array}{l}99.8 \\
(99.3)\end{array}$ & $\begin{array}{l}27.47 \\
(8.40)\end{array}$ & $\begin{array}{l}5.2 \\
(22.6)\end{array}$ & 27.58 & 79.1 & 37.9 & 0.163 & 99.9 & 0.91 \\
\hline 0.93 & 8550 & $\begin{array}{l}99.9 \\
(99.5)\end{array}$ & $\begin{array}{l}25.62 \\
(6.75)\end{array}$ & $\begin{array}{l}5.5 \\
(28.8)\end{array}$ & 29.46 & 79.2 & 37.9 & 0.167 & 99.9 & 0.81 \\
\hline 1.24 & 8567 & $\begin{array}{l}99.9 \\
(99.7)\end{array}$ & $\begin{array}{l}23.09 \\
(4.75)\end{array}$ & $\begin{array}{l}6.2 \\
(42.1)\end{array}$ & 32.31 & 79.2 & 37.9 & 0.172 & 99.9 & 0.68 \\
\hline 1.55 & 8587 & $\begin{array}{l}99.9 \\
(99.7)\end{array}$ & $\begin{array}{l}19.52 \\
(2.58)\end{array}$ & $\begin{array}{l}7.6 \\
(77.8)\end{array}$ & 36.87 & 79.3 & 37.9 & 0.187 & 99.9 & 0.51 \\
\hline 1.86 & 8594 & $\begin{array}{l}99.9 \\
(99.7)\end{array}$ & $\begin{array}{l}14.05 \\
(0.88)\end{array}$ & $\begin{array}{l}11.8 \\
(231.9)\end{array}$ & 44.94 & 79.4 & 38.0 & 0.226 & 99.9 & 0.32 \\
\hline 2.17 & 8425 & $\begin{array}{l}97.4 \\
(91.6)\end{array}$ & $\begin{array}{l}5.88 \\
(-)\end{array}$ & $\begin{array}{l}41.9 \\
(-)\end{array}$ & 58.71 & 79.5 & 38.0 & 0.477 & 99.1 & 0.18 \\
\hline
\end{tabular}

Crystal 12 (No uridine)

\begin{tabular}{|c|c|c|c|c|c|c|c|c|c|c|}
\hline $\begin{array}{l}\text { Dose } \\
\text { (MGy) }\end{array}$ & $\begin{array}{l}\text { Unique } \\
\text { reflections }\end{array}$ & $\begin{array}{l}\text { Complete } \\
\text {-ness }(\%)\end{array}$ & $\langle\mathrm{I}\rangle / \sigma(\mathrm{I})$ & $\begin{array}{l}\text { R-meas } \\
(\%)\end{array}$ & $\begin{array}{l}\text { B-factor } \\
(\AA 2)\end{array}$ & $\begin{array}{l}a=b \\
(\AA)\end{array}$ & $\begin{array}{l}\mathrm{c} \\
(\AA)\end{array}$ & $\begin{array}{l}\text { Mosaicity } \\
\text { (deg) }\end{array}$ & $\mathrm{CC} 1 / 2$ & $\mathrm{I}_{\mathrm{n}} / \mathrm{I}_{0}$ \\
\hline $0.36^{\S}$ & 8586 & $\begin{array}{l}99.9 \\
(99.8)\end{array}$ & $\begin{array}{l}16.76 \\
(14.14)\end{array}$ & $\begin{array}{l}5.9 \\
(7.6)\end{array}$ & 21.52 & 79.3 & 37.9 & 0.035 & 99.4 & 1.00 \\
\hline $0.72^{\#}$ & 8579 & $\begin{array}{l}99.8 \\
(99.7)\end{array}$ & $\begin{array}{l}21.96 \\
(17.77)\end{array}$ & $\begin{array}{l}5.0 \\
(9.3)\end{array}$ & 26.80 & 79.3 & 37.9 & 0.04 & 99.7 & 0.82 \\
\hline $1.08^{*}$ & 8581 & $\begin{array}{l}99.8 \\
(99.7)\end{array}$ & $\begin{array}{l}26.94 \\
(8.71)\end{array}$ & $\begin{array}{l}5.0 \\
(26.2)\end{array}$ & 33.38 & 79.3 & 37.9 & 0.061 & 99.9 & 0.51 \\
\hline 1.44 & 8566 & $\begin{array}{l}99.8 \\
(99.9)\end{array}$ & $\begin{array}{l}13.67 \\
(2.23)\end{array}$ & $\begin{array}{l}12.4 \\
(118.9)\end{array}$ & 37.27 & 79.3 & 37.8 & 0.148 & 99.8 & 0.27 \\
\hline 1.8 & 8580 & $\begin{array}{l}99.7 \\
(99.3)\end{array}$ & $\begin{array}{l}7.94 \\
(1.21)\end{array}$ & $\begin{array}{l}21.3 \\
(216.0)\end{array}$ & 39.37 & 79.3 & 37.9 & 0.152 & 99.5 & 0.13 \\
\hline
\end{tabular}

$\S$ data set used for PDB ID 5L9J; \# data set used for PDB ID 5LA5; * data set used for PDB ID 5LA8. 
Crystal 13 (No uridine)

\begin{tabular}{|c|c|c|c|c|c|c|c|c|c|c|}
\hline $\begin{array}{l}\text { Dose } \\
\text { (MGy) }\end{array}$ & $\begin{array}{l}\text { Unique } \\
\text { reflections }\end{array}$ & $\begin{array}{l}\text { Complete } \\
\text {-ness }(\%)\end{array}$ & $\langle\mathrm{I}\rangle / \sigma(\mathrm{I})$ & $\begin{array}{l}\text { R-meas } \\
(\%)\end{array}$ & $\begin{array}{l}\text { B-factor } \\
\text { (§2) }\end{array}$ & $\begin{array}{l}a=b \\
(\AA)\end{array}$ & $\begin{array}{l}\mathrm{c} \\
(\AA)\end{array}$ & $\begin{array}{l}\text { Mosaicity } \\
\text { (deg) }\end{array}$ & $\mathrm{CC} 1 / 2$ & $\mathrm{I}_{\mathrm{n}} / \mathrm{I}_{0}$ \\
\hline 0.36 & 8563 & $\begin{array}{l}99.7 \\
(99.6)\end{array}$ & $\begin{array}{l}42.67 \\
(34.65)\end{array}$ & $\begin{array}{l}3.8 \\
(5.2)\end{array}$ & 21.79 & 79.3 & 37.9 & 0.057 & 99.8 & 1.00 \\
\hline 0.72 & 8507 & $\begin{array}{l}99.0 \\
(98.4)\end{array}$ & $\begin{array}{l}46.17 \\
(38.02)\end{array}$ & $\begin{array}{l}3.5 \\
(5.4)\end{array}$ & 25.99 & 79.3 & 37.9 & 0.06 & 99.8 & 0.88 \\
\hline 1.08 & 8577 & $\begin{array}{l}99.8 \\
(100)\end{array}$ & $\begin{array}{l}34.92 \\
(14.45)\end{array}$ & $\begin{array}{l}3.8 \\
(15.2)\end{array}$ & 31.16 & 79.3 & 37.9 & 0.072 & 99.9 & 0.55 \\
\hline 1.44 & 8586 & $\begin{array}{l}99.8 \\
(99.5)\end{array}$ & $\begin{array}{l}24.7 \\
(5.33)\end{array}$ & $\begin{array}{l}6.4 \\
(47.9)\end{array}$ & 33.53 & 79.3 & 37.9 & 0.132 & 99.9 & 0.34 \\
\hline 1.8 & 8589 & $\begin{array}{l}99.7 \\
(99.0)\end{array}$ & $\begin{array}{l}14.22 \\
(2.09)\end{array}$ & $\begin{array}{l}12.1 \\
(110.7)\end{array}$ & 35.868 & 79.3 & 37.835 & 0.185 & 99.8 & 0.21 \\
\hline
\end{tabular}

Crystal 14 (No uridine)

\begin{tabular}{|c|c|c|c|c|c|c|c|c|c|c|}
\hline $\begin{array}{l}\begin{array}{l}\text { Dose } \\
\text { (MGy) }\end{array} \\
\end{array}$ & $\begin{array}{l}\text { Unique } \\
\text { reflections }\end{array}$ & $\begin{array}{l}\text { Complete } \\
\text {-ness }(\%)\end{array}$ & $\langle\mathrm{I}\rangle / \sigma(\mathrm{I})$ & $\begin{array}{l}\text { R-meas } \\
(\%)\end{array}$ & $\begin{array}{l}\text { B-factor } \\
(\AA 2)\end{array}$ & $\begin{array}{l}a=b \\
(\AA)\end{array}$ & $\begin{array}{l}\mathrm{c} \\
(\AA)\end{array}$ & $\begin{array}{l}\text { Mosaicity } \\
\text { (deg) }\end{array}$ & $\mathrm{CC} 1 / 2$ & $\mathrm{I}_{\mathrm{n}} / \mathrm{I}_{0}$ \\
\hline 0.36 & 8122 & $\begin{array}{l}94.6 \\
(92.1)\end{array}$ & $\begin{array}{l}33.64 \\
(29.71)\end{array}$ & $\begin{array}{l}4.8 \\
(6.2)\end{array}$ & 22.08 & 79.2 & 38.0 & 0.054 & 99.7 & 1.00 \\
\hline 0.72 & 8159 & $\begin{array}{l}94.9 \\
(92.5)\end{array}$ & $\begin{array}{l}33.86 \\
(16.93)\end{array}$ & $\begin{array}{l}3.9 \\
(11.0)\end{array}$ & 28.14 & 79.3 & 37.9 & 0.067 & 99.9 & 0.78 \\
\hline 1.08 & 8133 & $\begin{array}{l}94.6 \\
(92.1)\end{array}$ & $\begin{array}{l}28.93 \\
(6.55)\end{array}$ & $\begin{array}{l}4.9 \\
(34.2)\end{array}$ & 33.25 & 79.3 & 37.9 & 0.114 & 100 & 0.49 \\
\hline 1.44 & 8089 & $\begin{array}{l}94.0 \\
(91.2)\end{array}$ & $\begin{array}{l}15.13 \\
(1.61)\end{array}$ & $\begin{array}{l}12.2 \\
(143.1)\end{array}$ & 37.57 & 79.3 & 37.9 & 0.257 & 99.8 & 0.28 \\
\hline 1.8 & 8042 & $\begin{array}{l}93.6 \\
(91.8)\end{array}$ & $\begin{array}{l}9.13 \\
(0.7)\end{array}$ & $\begin{array}{l}21.7 \\
(291.2)\end{array}$ & 39.54 & 79.3 & 37.8 & 0.282 & 99.6 & 0.15 \\
\hline
\end{tabular}

Crystal 15 (No uridine)

\begin{tabular}{|c|c|c|c|c|c|c|c|c|c|c|}
\hline $\begin{array}{l}\text { Dose } \\
\text { (MGy) }\end{array}$ & $\begin{array}{l}\text { Unique } \\
\text { reflections }\end{array}$ & $\begin{array}{l}\text { Complete } \\
\text {-ness (\%) }\end{array}$ & $\langle\mathrm{I}\rangle / \sigma(\mathrm{I})$ & $\begin{array}{l}\text { R-meas } \\
(\%)\end{array}$ & $\begin{array}{l}\text { B-factor } \\
(\AA 2)\end{array}$ & $\begin{array}{l}\mathrm{a}=\mathrm{b} \\
(\AA)\end{array}$ & $\begin{array}{l}\mathrm{c} \\
(\AA)\end{array}$ & $\begin{array}{l}\text { Mosaicity } \\
(\mathrm{deg})\end{array}$ & $\mathrm{CC} 1 / 2$ & $\mathrm{I}_{\mathrm{n}} / \mathrm{I}_{0}$ \\
\hline 0.36 & 8526 & $\begin{array}{l}99.2 \\
(99.4)\end{array}$ & $\begin{array}{l}40.09 \\
(35.26)\end{array}$ & $\begin{array}{l}4.1 \\
(4.8)\end{array}$ & 21.25 & 79.2 & 37.9 & 0.053 & 99.7 & 1.00 \\
\hline 0.72 & 8570 & $\begin{array}{l}99.7 \\
(99.6)\end{array}$ & $\begin{array}{l}37.71 \\
(22.58)\end{array}$ & $\begin{array}{l}3.6 \\
(7.6)\end{array}$ & 26.86 & 79.3 & 37.9 & 0.057 & 99.9 & 0.80 \\
\hline 1.08 & 8585 & $\begin{array}{l}99.9 \\
(99.8)\end{array}$ & $\begin{array}{l}34.8 \\
(12.87)\end{array}$ & $\begin{array}{l}3.7 \\
(16.6)\end{array}$ & 32.25 & 79.3 & 37.9 & 0.081 & 99.9 & 0.52 \\
\hline 1.44 & 8569 & $\begin{array}{l}99.8 \\
(99.7)\end{array}$ & $\begin{array}{l}24.81 \\
(4.73)\end{array}$ & $\begin{array}{l}6.5 \\
(55.6)\end{array}$ & 35.50 & 79.3 & 37.9 & 0.152 & 99.9 & 0.29 \\
\hline 1.8 & 8535 & $\begin{array}{l}99.8 \\
(99.6)\end{array}$ & $\begin{array}{l}14.96 \\
(1.86)\end{array}$ & $\begin{array}{l}11.9 \\
(127.7)\end{array}$ & 38.99 & 79.3 & 37.9 & 0.223 & 99.8 & 0.16 \\
\hline
\end{tabular}

Crystal 16 (1M uridine)

\begin{tabular}{|c|c|c|c|c|c|c|c|c|c|c|}
\hline $\begin{array}{l}\text { Dose } \\
\text { (MGy) }\end{array}$ & $\begin{array}{l}\text { Unique } \\
\text { reflections }\end{array}$ & $\begin{array}{l}\text { Complete } \\
\text {-ness (\%) }\end{array}$ & $\langle\mathrm{I}\rangle / \sigma(\mathrm{I})$ & $\begin{array}{l}\text { R-meas } \\
(\%)\end{array}$ & $\begin{array}{l}\text { B-factor } \\
(\AA 2)\end{array}$ & $\begin{array}{l}\mathrm{a}=\mathrm{b} \\
(\AA)\end{array}$ & $\begin{array}{l}\mathrm{c} \\
(\AA)\end{array}$ & $\begin{array}{l}\text { Mosaicity } \\
(\mathrm{deg})\end{array}$ & $\mathrm{CC} 1 / 2$ & $\mathrm{I}_{\mathrm{n}} / \mathrm{I}_{0}$ \\
\hline $0.36^{\S}$ & 8525 & $\begin{array}{l}99.6 \\
(99.7)\end{array}$ & $\begin{array}{l}44.45 \\
(26.75)\end{array}$ & $\begin{array}{l}3.1 \\
(6.1)\end{array}$ & 23.59 & 79.1 & 37.9 & 0.058 & 99.9 & 1.00 \\
\hline $0.72^{\#}$ & 8523 & $\begin{array}{l}99.4 \\
(98.8)\end{array}$ & $\begin{array}{l}42.24 \\
(23.52)\end{array}$ & $\begin{array}{l}3.2 \\
(6.8)\end{array}$ & 25.46 & 79.1 & 37.9 & 0.063 & 99.9 & 0.92 \\
\hline $1.08^{*}$ & 8544 & $\begin{array}{l}99.7 \\
(99.9)\end{array}$ & $\begin{array}{l}42.53 \\
(20.74)\end{array}$ & $\begin{array}{l}3.0 \\
(8.0)\end{array}$ & 27.81 & 79.2 & 37.9 & 0.069 & 100 & 0.84 \\
\hline 1.44 & 8547 & $\begin{array}{l}99.7 \\
(99.6)\end{array}$ & $\begin{array}{l}41.21 \\
(16.04)\end{array}$ & $\begin{array}{l}3.1 \\
(11.0)\end{array}$ & 30.68 & 79.2 & 37.9 & 0.082 & 100 & 0.71 \\
\hline 1.8 & 8535 & $\begin{array}{l}99.6 \\
(99.7)\end{array}$ & $\begin{array}{l}35.37 \\
(8.90)\end{array}$ & $\begin{array}{l}3.7 \\
(22.1)\end{array}$ & 34.62 & 79.2 & 37.9 & 0.145 & 100 & 0.52 \\
\hline 2.16 & 8518 & $\begin{array}{l}99.5 \\
(99.7)\end{array}$ & $\begin{array}{l}24.99 \\
(4.21)\end{array}$ & $\begin{array}{l}6.0 \\
(48.8)\end{array}$ & 36.82 & 79.2 & 37.9 & 0.243 & 99.9 & 0.36 \\
\hline
\end{tabular}




\begin{tabular}{lllllllllll}
2.52 & 8475 & 99.3 & 16.16 & 9.1 & 39.12 & 79.1 & 37.8 & 0.323 & 99.9 & 0.23 \\
& & $(98.6)$ & $(2.15)$ & $(91.4)$ & & & & & & \\
2.88 & 8477 & 99.6 & 10.5 & 14.4 & 40.38 & 79.0 & 37.8 & 0.427 & 99.7 & 0.16 \\
& & $(99.7)$ & $(1.15)$ & $(167.7)$ & & & & & & \\
3.24 & 8319 & 99.0 & 5.65 & 23.4 & 39.90 & 78.7 & 37.6 & 0.391 & 99.1 & 0.10 \\
& & $(98.9)$ & $(0.85)$ & $(261.7)$ & & & & & & \\
\hline
\end{tabular}

$\S$ data set used for PDB ID 5LAF; \# data set used for PDB ID 5LAG; * data set used for PDB ID 5LAN.

Crystal 17 (1M uridine)

\begin{tabular}{|c|c|c|c|c|c|c|c|c|c|c|}
\hline $\begin{array}{l}\text { Dose } \\
\text { (MGy) }\end{array}$ & $\begin{array}{l}\text { Unique } \\
\text { reflections }\end{array}$ & $\begin{array}{l}\text { Complete } \\
\text {-ness }(\%)\end{array}$ & $\langle\mathrm{I}\rangle / \sigma(\mathrm{I})$ & $\begin{array}{l}\text { R-meas } \\
(\%)\end{array}$ & $\begin{array}{l}\text { B-factor } \\
(\AA 2)\end{array}$ & $\begin{array}{l}\mathrm{a}=\mathrm{b} \\
(\AA)\end{array}$ & $\begin{array}{l}\mathrm{c} \\
(\AA)\end{array}$ & $\begin{array}{l}\text { Mosaicity } \\
\text { (deg) }\end{array}$ & $\mathrm{CC} 1 / 2$ & $\mathrm{I}_{\mathrm{n}} / \mathrm{I}_{0}$ \\
\hline 0.36 & 8539 & $\begin{array}{l}99.7 \\
(99.7)\end{array}$ & $\begin{array}{l}39.0 \\
(22.78)\end{array}$ & $\begin{array}{l}3.5 \\
(7.2)\end{array}$ & 23.71 & 79.0 & 38.0 & 0.052 & 99.9 & 1.00 \\
\hline 0.72 & 8544 & $\begin{array}{l}99.7 \\
(99.8)\end{array}$ & $\begin{array}{l}37.38 \\
(20.56)\end{array}$ & $\begin{array}{l}3.6 \\
(8.2)\end{array}$ & 25.18 & 79.0 & 38.0 & 0.051 & 99.9 & 0.91 \\
\hline 1.08 & 8565 & $\begin{array}{l}99.9 \\
(99.9)\end{array}$ & $\begin{array}{l}36.06 \\
(16.20)\end{array}$ & $\begin{array}{l}3.6 \\
(10.4)\end{array}$ & 28.10 & 79.1 & 37.9 & 0.06 & 99.9 & 0.83 \\
\hline 1.44 & 8561 & $\begin{array}{l}99.8 \\
(99.8)\end{array}$ & $\begin{array}{l}34.44 \\
(12.04)\end{array}$ & $\begin{array}{l}3.7 \\
(14.7)\end{array}$ & 30.95 & 79.2 & 37.9 & 0.08 & 100 & 0.70 \\
\hline 1.8 & 8548 & $\begin{array}{l}99.8 \\
(99.5)\end{array}$ & $\begin{array}{l}29.91 \\
(7.87)\end{array}$ & $\begin{array}{l}4.3 \\
(24.5)\end{array}$ & 33.06 & 79.2 & 37.9 & 0.11 & 100 & 0.55 \\
\hline 2.16 & 8532 & $\begin{array}{l}99.8 \\
(100)\end{array}$ & $\begin{array}{l}24.06 \\
(4.68)\end{array}$ & $\begin{array}{l}5.9 \\
(45.1)\end{array}$ & 34.15 & 79.2 & 37.9 & 0.15 & 99.9 & 0.44 \\
\hline 2.52 & 8566 & $\begin{array}{l}99.7 \\
(99.3)\end{array}$ & $\begin{array}{l}18.78 \\
(2.73)\end{array}$ & $\begin{array}{l}8.6 \\
(77.3)\end{array}$ & 34.694 & 79.203 & 37.884 & 0.166 & 99.9 & 0.37 \\
\hline 2.88 & 8555 & $\begin{array}{l}99.7 \\
(99.0)\end{array}$ & $\begin{array}{l}14.63 \\
(1.78)\end{array}$ & $\begin{array}{l}11.8 \\
(118.1)\end{array}$ & 35.808 & 79.239 & 37.886 & 0.174 & 99.8 & 0.28 \\
\hline 3.24 & 8557 & $\begin{array}{l}99.7 \\
(99.0)\end{array}$ & $\begin{array}{l}10.56 \\
(1.21)\end{array}$ & $\begin{array}{l}16.9 \\
(172.2)\end{array}$ & 36.823 & 79.302 & 37.904 & 0.183 & 99.7 & 0.21 \\
\hline
\end{tabular}

Crystal 18 (1M uridine)

\begin{tabular}{|c|c|c|c|c|c|c|c|c|c|c|}
\hline $\begin{array}{l}\text { Dose } \\
\text { (MGy) }\end{array}$ & $\begin{array}{l}\text { Unique } \\
\text { reflections }\end{array}$ & $\begin{array}{l}\text { Complete } \\
\text {-ness (\%) }\end{array}$ & $\langle\mathrm{I}\rangle / \sigma(\mathrm{I})$ & $\begin{array}{l}\text { R-meas } \\
(\%)\end{array}$ & $\begin{array}{l}\text { B-factor } \\
(\AA 2)\end{array}$ & $\begin{array}{l}\mathrm{a}=\mathrm{b} \\
(\AA)\end{array}$ & $\begin{array}{l}\mathrm{c} \\
(\AA)\end{array}$ & $\begin{array}{l}\text { Mosaicity } \\
(\mathrm{deg})\end{array}$ & $\mathrm{CC} 1 / 2$ & $\mathrm{I}_{\mathrm{n}} / \mathrm{I}_{0}$ \\
\hline 0.36 & 8552 & $\begin{array}{l}99.7 \\
(99.9)\end{array}$ & $\begin{array}{l}18.49 \\
(14.2)\end{array}$ & $\begin{array}{l}4.9 \\
(7.8)\end{array}$ & 22.22 & 79.1 & 37.9 & 0.032 & 99.6 & 1.00 \\
\hline 0.72 & 8543 & $\begin{array}{l}99.6 \\
(99.7)\end{array}$ & $\begin{array}{l}38.58 \\
(21.32)\end{array}$ & $\begin{array}{l}3.4 \\
(8.3)\end{array}$ & 24.91 & 79.2 & 37.9 & 0.039 & 99.9 & 0.95 \\
\hline 1.08 & 8557 & $\begin{array}{l}99.7 \\
(99.9)\end{array}$ & $\begin{array}{l}36.47 \\
(16.96)\end{array}$ & $\begin{array}{l}3.5 \\
(10.0)\end{array}$ & 27.52 & 79.2 & 37.9 & 0.054 & 99.9 & 0.88 \\
\hline 1.44 & 8567 & $\begin{array}{l}99.7 \\
(99.3)\end{array}$ & $\begin{array}{l}34.25 \\
(11.97)\end{array}$ & $\begin{array}{l}3.8 \\
(16.3)\end{array}$ & 30.65 & 79.2 & 37.9 & 0.078 & 100 & 0.76 \\
\hline 1.8 & 8549 & $\begin{array}{l}99.7 \\
(99.6)\end{array}$ & $\begin{array}{l}28.13 \\
(6.68)\end{array}$ & $\begin{array}{l}5.0 \\
(33.4)\end{array}$ & 33.66 & 79.2 & 37.9 & 0.13 & 99.9 & 0.60 \\
\hline 2.16 & 8538 & $\begin{array}{l}99.6 \\
(99.3)\end{array}$ & $\begin{array}{l}19.29 \\
(3.03)\end{array}$ & $\begin{array}{l}8.3 \\
(75.9)\end{array}$ & 36.16 & 79.2 & 37.9 & 0.18 & 99.9 & 0.46 \\
\hline 2.52 & 8550 & $\begin{array}{l}99.6 \\
(99.3)\end{array}$ & $\begin{array}{l}13.36 \\
(1.80)\end{array}$ & $\begin{array}{l}13.4 \\
(131.1)\end{array}$ & 37.37 & 79.2 & 37.9 & 0.213 & 99.8 & 0.35 \\
\hline 2.88 & 8533 & $\begin{array}{l}99.3 \\
(98.1)\end{array}$ & $\begin{array}{l}9.86 \\
(1.19)\end{array}$ & $\begin{array}{l}19.7 \\
(200.1)\end{array}$ & 38.60 & 79.2 & 37.9 & 0.224 & 99.5 & 0.26 \\
\hline 3.24 & 8565 & $\begin{array}{l}99.3 \\
(98.0)\end{array}$ & $\begin{array}{l}7.56 \\
(0.86)\end{array}$ & $\begin{array}{l}26.0 \\
(280.0)\end{array}$ & 39.29 & 79.2 & 37.9 & 0.213 & 99.2 & 0.19 \\
\hline
\end{tabular}


Table S3 Data collection statistics for all crystals collected at $100 \mathrm{~K}$ extracted from the initial integration by XDS. Resolution range is 50-1.7 $\AA$. Space group for all crystals is $\mathrm{P} 4_{3} 22_{1}$.

Crystal 18 (No uridine)

\begin{tabular}{|c|c|c|c|c|c|c|c|c|c|c|}
\hline $\begin{array}{l}\text { Dose } \\
\text { (MGy) }\end{array}$ & $\begin{array}{l}\text { Unique } \\
\text { reflections }\end{array}$ & $\begin{array}{l}\text { Complete } \\
\text {-ness }(\%)\end{array}$ & $\langle\mathrm{I}\rangle / \sigma(\mathrm{I})$ & $\begin{array}{l}\text { R-meas } \\
(\%)\end{array}$ & $\begin{array}{l}\text { B-factor } \\
\left(\AA^{2}\right)\end{array}$ & $\begin{array}{l}\mathrm{a}=\mathrm{b} \\
(\AA)\end{array}$ & $\begin{array}{l}\mathrm{c} \\
(\AA)\end{array}$ & $\begin{array}{l}\text { Mosaicity } \\
(\mathrm{deg})\end{array}$ & $\mathrm{CC} 1 / 2$ & $\mathrm{In} / \mathrm{IO}$ \\
\hline 1.61 & 13288 & $\begin{array}{l}99.7 \\
(99.6)\end{array}$ & $\begin{array}{l}66.15 \\
(36.44)\end{array}$ & $\begin{array}{l}2.1 \\
(4.3)\end{array}$ & 17.43 & 78.8 & 37.0 & 0.181 & 100.0 & 1.00 \\
\hline 45.93 & 13366 & $\begin{array}{l}99.6 \\
(99.2)\end{array}$ & $\begin{array}{l}50.85 \\
(21.29)\end{array}$ & $\begin{array}{l}2.5 \\
(7.5)\end{array}$ & 21.94 & 79.0 & 37.1 & 0.174 & 100.0 & 0.66 \\
\hline 101.33 & 13415 & $\begin{array}{l}99.6 \\
(99.5)\end{array}$ & $\begin{array}{l}27.6 \\
(6.05)\end{array}$ & $\begin{array}{l}4.1 \\
(27.1)\end{array}$ & 29.33 & 79.0 & 37.1 & 0.189 & 99.9 & 0.37 \\
\hline 145.65 & 13345 & $\begin{array}{l}99.5 \\
(99.3)\end{array}$ & $\begin{array}{l}17.96 \\
(2.21)\end{array}$ & $\begin{array}{l}5.8 \\
(79.9)\end{array}$ & 34.70 & 79.1 & 37.1 & 0.209 & 99.9 & 0.23 \\
\hline
\end{tabular}

Crystal 19 (No uridine)

\begin{tabular}{|c|c|c|c|c|c|c|c|c|c|c|}
\hline $\begin{array}{l}\begin{array}{l}\text { Dose } \\
\text { (MGy) }\end{array} \\
\end{array}$ & $\begin{array}{l}\text { Unique } \\
\text { reflections }\end{array}$ & $\begin{array}{l}\text { Complete } \\
\text {-ness (\%) }\end{array}$ & $\langle\mathrm{I}\rangle / \sigma(\mathrm{I})$ & $\begin{array}{l}\text { R-meas } \\
(\%)\end{array}$ & $\begin{array}{l}\text { B-factor } \\
\left(\AA^{2}\right)\end{array}$ & $\begin{array}{l}a=b \\
(\AA)\end{array}$ & $\begin{array}{l}\mathrm{c} \\
(\AA)\end{array}$ & $\begin{array}{l}\text { Mosaicity } \\
\text { (deg) }\end{array}$ & $\mathrm{CC} 1 / 2$ & In / I0 \\
\hline 1.61 & 12646 & $\begin{array}{l}95.0 \\
(97.1)\end{array}$ & $\begin{array}{l}72.99 \\
(39.44)\end{array}$ & $\begin{array}{l}1.9 \\
(3.9)\end{array}$ & 17.84 & 78.7 & 37.0 & 0.156 & 100.0 & 1.00 \\
\hline 45.93 & 12695 & $\begin{array}{l}95.0 \\
(97.5)\end{array}$ & $\begin{array}{l}56.91 \\
(20.03)\end{array}$ & $\begin{array}{l}2.1 \\
(7.8)\end{array}$ & 22.65 & 78.9 & 37.1 & 0.181 & 100.0 & 0.69 \\
\hline 101.33 & 12579 & $\begin{array}{l}94.5 \\
(95.7)\end{array}$ & $\begin{array}{l}28.53 \\
(4.85)\end{array}$ & $\begin{array}{l}3.8 \\
(33.1)\end{array}$ & 30.56 & 78.8 & 37.1 & 0.245 & 100.0 & 0.40 \\
\hline 145.65 & 12473 & $\begin{array}{l}94.3 \\
(95.4)\end{array}$ & $\begin{array}{l}17.75 \\
(1.86)\end{array}$ & $\begin{array}{l}5.8 \\
(89.7)\end{array}$ & 34.57 & 78.7 & 37.1 & 0.297 & 99.9 & 0.25 \\
\hline
\end{tabular}

Crystal 20 (No uridine)

\begin{tabular}{|c|c|c|c|c|c|c|c|c|c|c|}
\hline $\begin{array}{l}\begin{array}{l}\text { Dose } \\
\text { (MGy) }\end{array} \\
\end{array}$ & $\begin{array}{l}\text { Unique } \\
\text { reflections }\end{array}$ & $\begin{array}{l}\text { Complete } \\
\text {-ness }(\%)\end{array}$ & $\langle\mathrm{I}\rangle / \sigma(\mathrm{I})$ & $\begin{array}{l}\text { R-meas } \\
(\%)\end{array}$ & $\begin{array}{l}\text { B-factor } \\
\left(\AA^{2}\right)\end{array}$ & $\begin{array}{l}\mathrm{a}=\mathrm{b} \\
(\AA)\end{array}$ & $\begin{array}{l}\mathrm{c} \\
(\AA)\end{array}$ & $\begin{array}{l}\text { Mosaicity } \\
\text { (deg) }\end{array}$ & $\mathrm{CC} 1 / 2$ & In / IC \\
\hline 1.61 & 13255 & $\begin{array}{l}99.7 \\
(99.3)\end{array}$ & $\begin{array}{l}59.78 \\
(36.77)\end{array}$ & $\begin{array}{l}2.5 \\
(4.3)\end{array}$ & 16.36 & 78.8 & 37.0 & 0.113 & 99.9 & 1.00 \\
\hline 45.93 & 13379 & $\begin{array}{l}99.8 \\
(99.9)\end{array}$ & $\begin{array}{l}55.62 \\
(19.44)\end{array}$ & $\begin{array}{l}2.2 \\
(8.8)\end{array}$ & 21.13 & 79.0 & 37.1 & 0.134 & 100.0 & 0.74 \\
\hline 101.33 & 13386 & $\begin{array}{l}99.7 \\
(99.7)\end{array}$ & $\begin{array}{l}34.49 \\
(4.38)\end{array}$ & $\begin{array}{l}3.7 \\
(39.5)\end{array}$ & 28.74 & 79.1 & 37.1 & 0.164 & 100.0 & 0.44 \\
\hline 145.65 & 13417 & $\begin{array}{l}99.5 \\
(98.5) \\
\end{array}$ & $\begin{array}{l}23.60 \\
(1.59) \\
\end{array}$ & $\begin{array}{l}5.5 \\
(124.9) \\
\end{array}$ & 34.43 & 79.2 & 37.1 & 0.188 & 100.0 & 0.28 \\
\hline
\end{tabular}

Crystal 21 (500mM uridine)

\begin{tabular}{|c|c|c|c|c|c|c|c|c|c|c|}
\hline $\begin{array}{l}\text { Dose } \\
\text { (MGy) }\end{array}$ & $\begin{array}{l}\text { Unique } \\
\text { reflections }\end{array}$ & $\begin{array}{l}\text { Complete } \\
\text {-ness }(\%)\end{array}$ & $\langle\mathrm{I}\rangle / \sigma(\mathrm{I})$ & $\begin{array}{l}\text { R-meas } \\
(\%)\end{array}$ & $\begin{array}{l}\text { B-factor } \\
\left(\AA^{2}\right)\end{array}$ & $\begin{array}{l}a=b \\
(\AA)\end{array}$ & $\begin{array}{l}\mathrm{c} \\
(\AA)\end{array}$ & $\begin{array}{l}\text { Mosaicity } \\
(\mathrm{deg})\end{array}$ & $\mathrm{CC} 1 / 2$ & In / I0 \\
\hline 1.61 & 13265 & $\begin{array}{l}99.7 \\
(99.0)\end{array}$ & $\begin{array}{l}48.02 \\
(34.84)\end{array}$ & $\begin{array}{l}3.4 \\
(4.6)\end{array}$ & 16.96 & 78.7 & 37.0 & 0.126 & 99.9 & 1.00 \\
\hline 45.93 & 13395 & $\begin{array}{l}99.8 \\
(99.6)\end{array}$ & $\begin{array}{l}47.64 \\
(19.92)\end{array}$ & $\begin{array}{l}2.8 \\
(8.2)\end{array}$ & 21.94 & 78.9 & 37.1 & 0.137 & 100.0 & 0.75 \\
\hline 101.33 & 13423 & $\begin{array}{l}99.8 \\
(99.1)\end{array}$ & $\begin{array}{l}30.83 \\
(4.36)\end{array}$ & $\begin{array}{l}3.9 \\
(45.3)\end{array}$ & 30.44 & 79.1 & 37.2 & 0.172 & 100.0 & 0.45 \\
\hline 145.65 & 13570 & $\begin{array}{l}99.8 \\
(99.3)\end{array}$ & $\begin{array}{l}19.36 \\
(1.19)\end{array}$ & $\begin{array}{l}5.9 \\
(165.1)\end{array}$ & 37.33 & 79.2 & 37.2 & 0.176 & 100.0 & 0.29 \\
\hline
\end{tabular}

Crystal 22 (1M uridine)

\begin{tabular}{lllllllllll}
\hline $\begin{array}{l}\text { Dose } \\
(\mathrm{MGy})\end{array}$ & $\begin{array}{l}\text { Unique } \\
\text { reflections }\end{array}$ & $\begin{array}{l}\text { Complete } \\
\text {-ness }(\%)\end{array}$ & $<\mathrm{I}>/ \sigma(\mathrm{I})$ & $\begin{array}{l}\text { R-meas } \\
(\%)\end{array}$ & $\begin{array}{l}\mathrm{B}-\text { factor } \\
\left(\AA^{2}\right)\end{array}$ & $\begin{array}{l}\mathrm{a}=\mathrm{b} \\
(\AA)\end{array}$ & $\begin{array}{l}\mathrm{c} \\
(\AA)\end{array}$ & $\begin{array}{l}\text { Mosaicity } \\
(\mathrm{deg})\end{array}$ & $\mathrm{CC} 1 / 2$ & $\mathrm{In} / \mathrm{I} 0$
\end{tabular}




\begin{tabular}{|c|c|c|c|c|c|c|c|c|c|c|}
\hline 1.61 & 13070 & $\begin{array}{l}99.6 \\
(99.6)\end{array}$ & $\begin{array}{l}48.60 \\
(15.04)\end{array}$ & $\begin{array}{l}2.7 \\
(11.6)\end{array}$ & 22.17 & 78.2 & 37.1 & 0.314 & 100.0 & 1.00 \\
\hline 45.93 & 13200 & $\begin{array}{l}99.7 \\
(99.6)\end{array}$ & $\begin{array}{l}40.05 \\
(8.28)\end{array}$ & $\begin{array}{l}3.1 \\
(22.2)\end{array}$ & 26.85 & 78.5 & 37.2 & 0.308 & 100.0 & 0.66 \\
\hline 101.33 & 13323 & $\begin{array}{l}99.7 \\
(99.6)\end{array}$ & $\begin{array}{l}24.45 \\
(2.01)\end{array}$ & $\begin{array}{l}4.6 \\
(98.1)\end{array}$ & 35.37 & 78.7 & 37.3 & 0.298 & 100.0 & 0.36 \\
\hline 145.65 & 13379 & $\begin{array}{l}99.5 \\
(98.1) \\
\end{array}$ & $\begin{array}{l}16.93 \\
(0.46) \\
\end{array}$ & $\begin{array}{l}6.6 \\
(445.7) \\
\end{array}$ & 43.58 & 78.7 & 37.3 & 0.335 & 100.0 & 0.22 \\
\hline
\end{tabular}

Crystal 23 (1M uridine)

\begin{tabular}{|c|c|c|c|c|c|c|c|c|c|c|}
\hline $\begin{array}{l}\begin{array}{l}\text { Dose } \\
\text { (MGy) }\end{array} \\
\end{array}$ & $\begin{array}{l}\text { Unique } \\
\text { reflections }\end{array}$ & $\begin{array}{l}\text { Complete } \\
\text {-ness }(\%)\end{array}$ & $\langle\mathrm{I}\rangle / \sigma(\mathrm{I})$ & $\begin{array}{l}\text { R-meas } \\
(\%)\end{array}$ & $\begin{array}{l}\text { B-factor } \\
\left(\AA^{2}\right)\end{array}$ & $\begin{array}{l}\mathrm{a}=\mathrm{b} \\
(\AA)\end{array}$ & $\begin{array}{l}\mathrm{c} \\
(\AA)\end{array}$ & $\begin{array}{l}\text { Mosaicity } \\
\text { (deg) }\end{array}$ & $\mathrm{CC} 1 / 2$ & In / I0 \\
\hline 1.61 & 13251 & $\begin{array}{l}99.7 \\
(99.8)\end{array}$ & $\begin{array}{l}50.96 \\
(27.63)\end{array}$ & $\begin{array}{l}2.8 \\
(5.9)\end{array}$ & 20.05 & 78.6 & 37.0 & 0.113 & 99.9 & 1.00 \\
\hline 45.93 & 13339 & $\begin{array}{l}99.7 \\
(99.9)\end{array}$ & $\begin{array}{l}42.13 \\
(16.32)\end{array}$ & $\begin{array}{l}3.1 \\
(10.4)\end{array}$ & 24.88 & 78.8 & 37.1 & 0.107 & 99.9 & 0.67 \\
\hline 101.33 & 13378 & $\begin{array}{l}99.7 \\
(99.6)\end{array}$ & $\begin{array}{l}30.16 \\
(4.63)\end{array}$ & $\begin{array}{l}3.7 \\
(41.1)\end{array}$ & 32.73 & 79.0 & 37.2 & 0.116 & 100.0 & 0.40 \\
\hline 145.65 & 13373 & $\begin{array}{l}99.5 \\
(99.2)\end{array}$ & $\begin{array}{l}21.78 \\
(1.32)\end{array}$ & $\begin{array}{l}4.9 \\
(152.1)\end{array}$ & 39.18 & 79.0 & 37.2 & 0.136 & 99.9 & 0.27 \\
\hline
\end{tabular}

Crystal 24 (1M uridine)

\begin{tabular}{|c|c|c|c|c|c|c|c|c|c|c|}
\hline $\begin{array}{l}\begin{array}{l}\text { Dose } \\
\text { (MGy) }\end{array} \\
\end{array}$ & $\begin{array}{l}\text { Unique } \\
\text { reflections }\end{array}$ & $\begin{array}{l}\text { Complete } \\
\text {-ness }(\%)\end{array}$ & $\langle\mathrm{I}\rangle / \sigma(\mathrm{I})$ & $\begin{array}{l}\text { R-meas } \\
(\%)\end{array}$ & $\begin{array}{l}\text { B-factor } \\
\left(\AA^{2}\right)\end{array}$ & $\begin{array}{l}\mathrm{a}=\mathrm{b} \\
(\AA)\end{array}$ & $\begin{array}{l}\mathrm{c} \\
(\AA)\end{array}$ & $\begin{array}{l}\text { Mosaicity } \\
(\mathrm{deg})\end{array}$ & $\mathrm{CC} 1 / 2$ & In / I0 \\
\hline 1.61 & 13247 & $\begin{array}{l}99.7 \\
(99.0)\end{array}$ & $\begin{array}{l}70.14 \\
(34.77)\end{array}$ & $\begin{array}{l}1.9 \\
(4.6)\end{array}$ & 19.53 & 78.6 & 37.0 & 0.145 & 100.0 & 1.00 \\
\hline 45.93 & 13338 & $\begin{array}{l}99.7 \\
(99.6)\end{array}$ & $\begin{array}{l}58.05 \\
(19.75)\end{array}$ & $\begin{array}{l}2.2 \\
(8.5)\end{array}$ & 23.84 & 78.8 & 37.2 & 0.140 & 100.0 & 0.71 \\
\hline 101.33 & 13341 & $\begin{array}{l}99.7 \\
(99.1)\end{array}$ & $\begin{array}{l}34.15 \\
(4.88)\end{array}$ & $\begin{array}{l}3.3 \\
(38.1)\end{array}$ & 31.17 & 78.9 & 37.2 & 0.167 & 100.0 & 0.43 \\
\hline 145.65 & 13340 & $\begin{array}{l}99.7 \\
(99.1)\end{array}$ & $\begin{array}{l}22.12 \\
(1.61)\end{array}$ & $\begin{array}{l}4.9 \\
(121.2)\end{array}$ & 36.66 & 78.9 & 37.2 & 0.195 & 100.0 & 0.28 \\
\hline
\end{tabular}




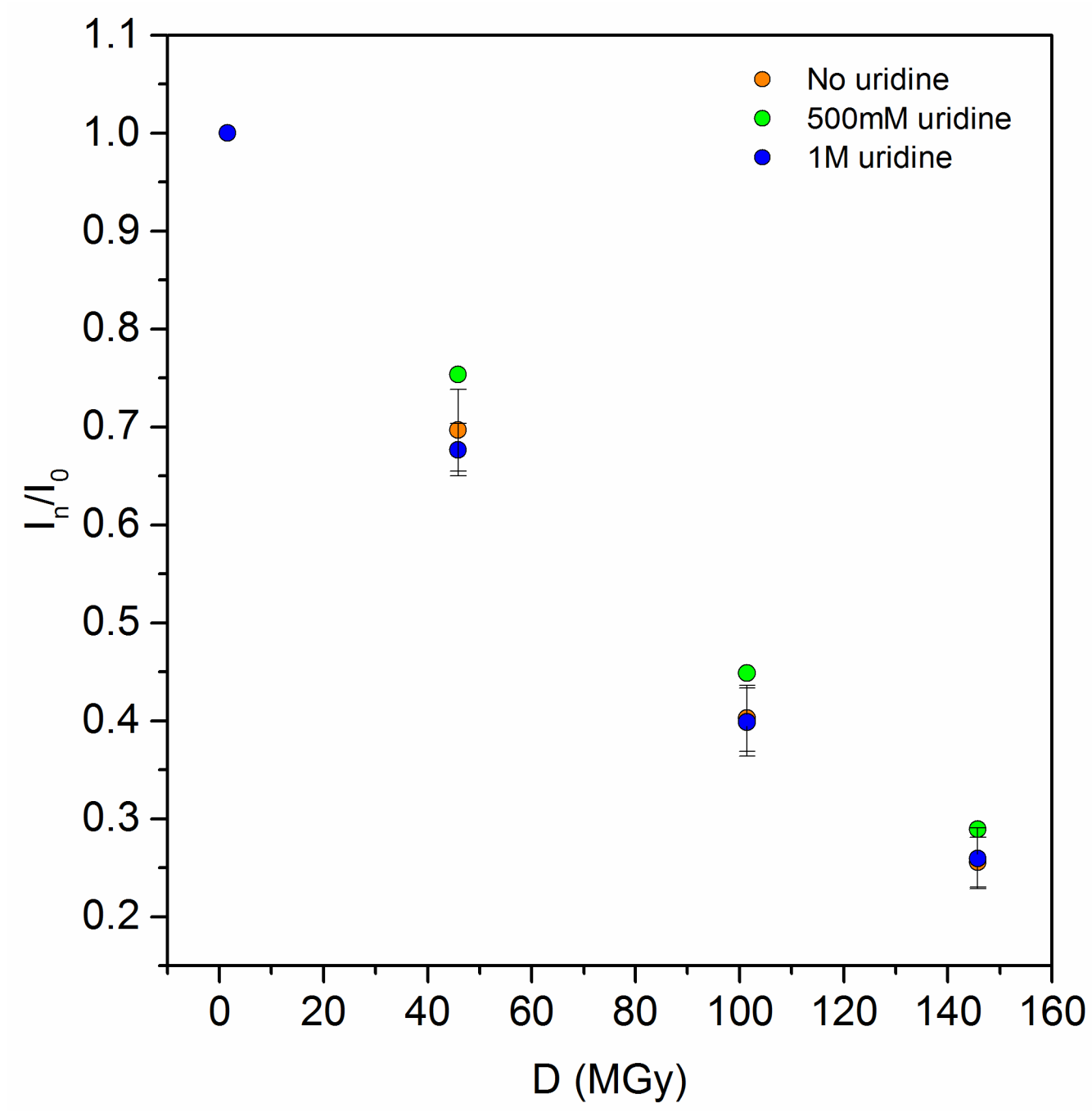

Figure S1 Decay of the mean normalized intensity $\left(\mathrm{I} / \mathrm{I}_{0}\right)$ with absorbed dose on lysozyme crystals at $100 \mathrm{~K}$ without uridine in the solution (orange, crystals 18-20), with $500 \mathrm{mM}$ uridine (green, crystal 21) and with $1 \mathrm{M}$ uridine (blue, crystals 22-24). $\mathrm{I} / \mathrm{I}_{0}$ is calculated in the 50-2 A resolution range. 

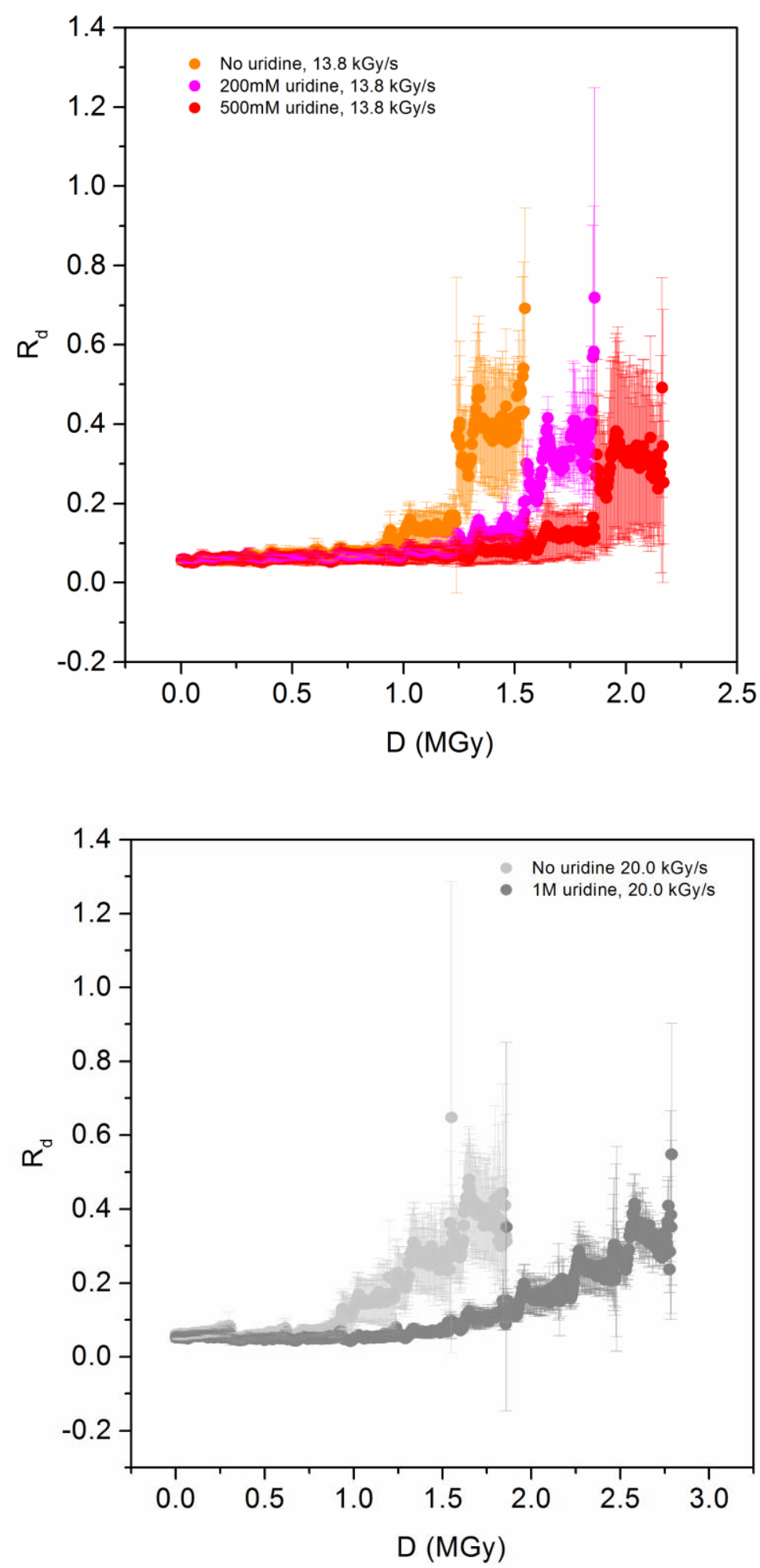

Figure S2 Evolution of the damage R-factor $\left(\mathrm{R}_{\mathrm{d}}\right)$ as a function of dose for native lysozyme crystals and lysozyme crystals soaked in different concentrations of uridine $(200 \mathrm{mM}, 500 \mathrm{mM}$ and $1 \mathrm{M})$. (Top) Data collected at $13.8 \mathrm{kGy} \mathrm{s}^{-1}$ and (bottom) data collected at $20.0 \mathrm{kGy} \mathrm{s}^{-1}$. $\mathrm{R}_{\mathrm{d}}$ is calculated in the resolution range 50-2 $\AA$. Each point in the plot is the mean obtained by 3 or 4 independent measures from different crystals. The associated error bar to each point shows the standard deviation of the mean. 


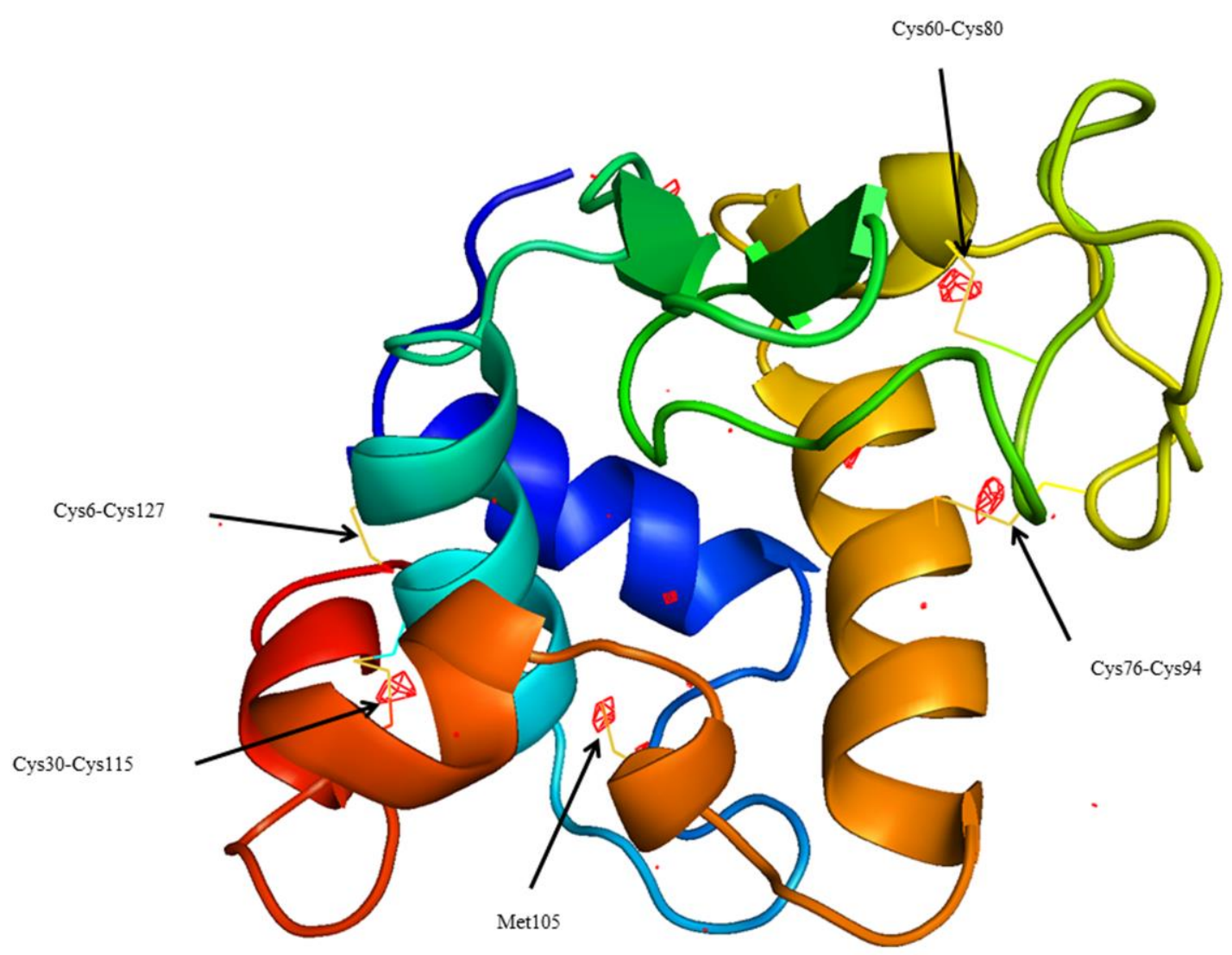

Figure S3 $\left(\mathrm{F}_{\mathrm{obs}, 3}-\mathrm{F}_{\mathrm{obs}, 1}, \alpha_{\mathrm{calc}, 1}\right)$ map of a crystal with $1 \mathrm{M}$ of uridine at room temperature (crystal 16 from table S2) contoured at $-4 \sigma$. The arrows point to the four disulphide bonds and to Met105.Only four relevant peaks are observed in Cys30-Cys115, Cys76-Cys94, Cys64-Cys80 and Met105. At -5б no significant peaks are observed. 


\section{S2. SAXS results}

Table S4 Comparison of beamline parameters and experimental conditions for different radiation damage studies performed for lysozyme at various SAXS beamlines. All the experiments were performed in $40 \mathrm{mM}$ sodium acetate, $\mathrm{pH} 3.8,150 \mathrm{mM} \mathrm{NaCl}$.

\begin{tabular}{llll}
\hline & $\begin{array}{l}\text { This study } \\
(\text { P12, PETRA III) }\end{array}$ & $\begin{array}{l}\text { Jeffries } \text { et al. 2015 } \\
(\text { P12, PETRA III) }\end{array}$ & $\begin{array}{l}\text { Kuwamoto } \text { et al. 2004 } \\
(\text { BL45XU, SPring-8) }\end{array}$ \\
Concentration $(\mathrm{mg} / \mathrm{ml})$ & 7.2 & 8.8 & 4.9 \\
Flux (photons/second) & $1.4 \times 10^{12}$ & $4 \times 10^{12}$ & $2.2 \times 10^{11}$ \\
Max. Beam size $(\mu \mathrm{m}, \mathrm{h} \times \mathrm{v})$ & $500 \times 250$ & $500 \times 250$ & $800 \times 600$ \\
Photon energy $(\mathrm{keV})$ & 10 & 10 & 13.8
\end{tabular}
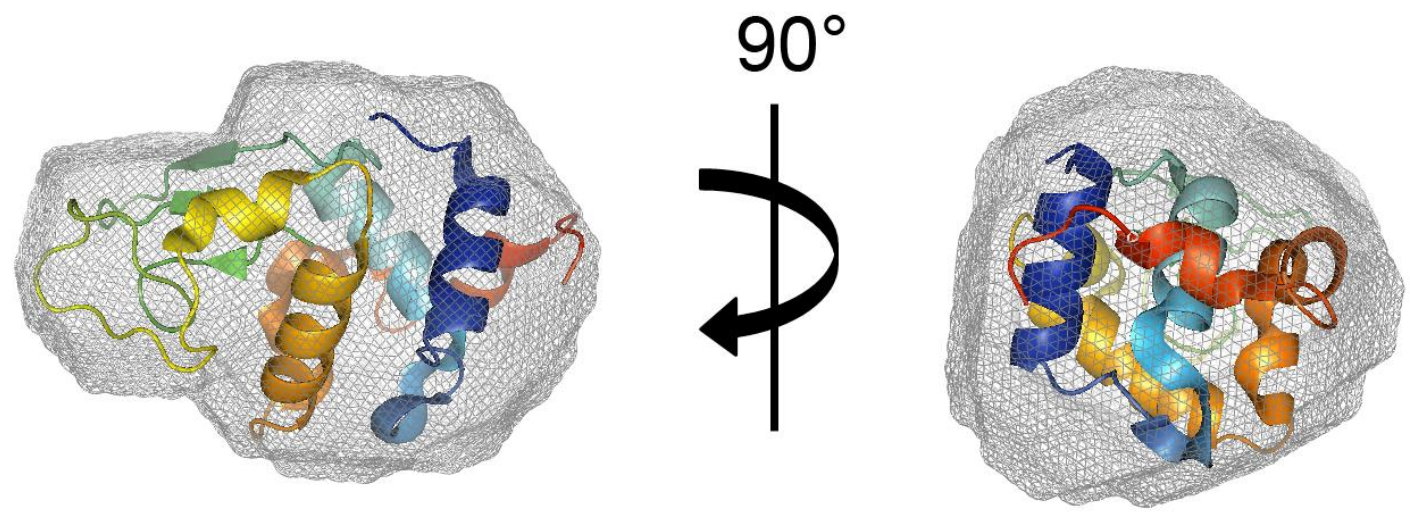

Figure S4 Ab initio reconstruction of the lysozyme low-resolution envelope from SAXS data collected in presence of $100 \mathrm{mM}$ uridine, obtained using DAMMIF (Franke \& Svergun, 2009) (grey mesh). The crystal structure of lysozyme (PDB ID: 4N8Z) was docked into the SAXS envelope by using SUPCOMB (Kozin \& Svergun, 2001) and rendered using PyMOL. The low-resolution model has a NSD value of 0.499 . 